\title{
Analysis of the IEEE 802.11e EDCA Under Statistical Traffic
}

\author{
Juki Wirawan Tantra, Chuan Heng Foh \\ Centre for Multimedia and Network Technology \\ School of Computer Engineering \\ Nanyang Technological University \\ Singapore \\ Email: jw.tantra@ieee.org, aschfoh@ntu.edu.sg
}

\author{
Ilenia Tinnirello \\ Dep. of Electrical Engineering \\ Università di Palermo \\ Palermo, Italy \\ Email: ilenia.tinnirello@tti.unipa.it
}

\author{
Giuseppe Bianchi \\ Dep. of Electrical Engineering \\ Università degli Studi di Roma \\ Tor Vergata \\ Roma, Italy \\ Email: bianchi@elet.polimi.it
}

\begin{abstract}
Many models have been proposed to analyze the performance of the IEEE 802.11 distributed coordination function (DCF) and the IEEE 802.11e enhanced distributed coordination function (EDCA) under saturation condition. To analyze DCF under statistical traffic, Foh and Zukerman introduce a model that uses Markovian Framework to compute the throughput and delay performance. In this paper, we analyze the protocol service time of EDCA mechanism and introduce a model to analyze EDCA under statistical traffic using Markovian Framework. Using this model, we analyze the throughput and delay performance of EDCA mechanism under statistical traffic.
\end{abstract}

\section{INTRODUCTION}

Widespread usage of high speed wireless local area networks (WLANs) has generated much interests on quality of service (QoS) support in WLANs. High speed WLANs allow users to run many bandwidth intensive applications without the hassle of wires. Nevertheless, supporting delay sensitive applications is difficult in WLANs as WLANs use unreliable medium. Most of the current WLAN devices implement the IEEE 802.11 standard [1], which uses contention based Carrier Sense Multiple Access with Collision Avoidance (CSMA/CA) as the main MAC protocol.

To support QoS demanding applications, IEEE is working on a new standard, the IEEE 802.11e [2], which specifies differentiated service classes in the MAC layer to support the delivery of priority packets such as multimedia data packets. IEEE 802.11e defines hybrid coordination function (HCF) for access mechanism. HCF itself uses two mechanisms for transmissions, which are enhanced distributed channel access (EDCA) and HCF controlled channel access (HCCA). EDCA is a contention based access mechanism, whereby HCCA is an optional polling based access mechanism. EDCA is basically an extension of the IEEE 802.11 distributed coordination function (DCF) mechanism, which implementes CSMA/CA, to support differentiated service classes.

Many models have been proposed to analyze the throughput and delay of IEEE 802.11 DCF mechanism and IEEE 802.11e EDCA mechanism. Bianchi [3] developed an analytical model to compute the saturation throughput of the stations that implement DCF mechanism. Using similar model, Chatzimisios et al. [4] derived the delay of packet transmissions. Robinson and Randhawa [5] extended Bianchi's model to analyze the saturation throughput performance of the EDCA mechanism. Tantra et al. [6] analyzed the throughput and delay performance of EDCA saturation. Foh and Zukerman [7] proposed a Markovian Framework model to analyze the performance of DCF under statistical traffic. In [8], Patil and Apte uses this Markovian Framework model to find the maximum number of users that can be supported by an Access Point.

In this paper, we analyze the protocol service time of EDCA mechanism and extend the model in [7] to analyze the EDCA mechanism. We then verify the model using simulations. Using this model, we analyze the throughput and delay performance of EDCA under statistical traffic. Our model is useful to predict the performance of a WLAN that implements EDCA. It can also be used for network sizing by extending the method in [8].

This paper is organized as follow. Section II provides a brief summary of the IEEE 802.11 DCF and IEEE 802.11e EDCA. In section III, we describe the saturation model that we use for the Markovian Framework. Section IV explains the variance analysis of EDCA that we developed for EDCA protocol analysis and for selection of parameters for our Markovian Framework model. Section V describes the Markovian Framework model that we developed to analyze the EDCF mechanism. We discuss and verify the results of our analytical model in section VI.

\section{MAC PROTOCOLS}

This section briefly summarizes the operations of the IEEE 802.11 DCF and the IEEE 802.11e EDCA. For a detailed description, readers may refer to [1] and [2].

\section{A. Distributed Coordination Function}

DCF employs CSMA/CA MAC protocol with binary exponential backoff. DCF does not use collision detection function as stations cannot detect collisions by listening to their own transmissions; thus, it employs handshaking method, which makes use of positive acknowledgment.

When a station generates a new frame for transmission, it will first monitor the channel activity. If the channel is detected idle for a period of time called DCF interframe space (DIFS), the station can transmit immediately. If the channel is 
busy, the station will defer until the end of transmission and a random backoff interval is selected. The backoff counter is decremented as long as the channel is sensed idle, stopped when channel activity is detected, and reactivated when the channel is sensed idle for more than a DIFS again. The station transmits its frame when the backoff counter reaches zero.

DCF uses a slotted binary exponential backoff technique. The period following an idle DIFS is slotted and the backoff time counter is measured in terms of slot time. The slot time is the time needed for any station to detect transmission from the other stations. It accounts for the propagation delay, the time needed to switch from the receiving to the transmitting state and the time to notify the MAC layer about the state of the channel.

The backoff time is uniformly chosen in the range $(0, C W$ $1)$, where $C W$ is the current contention window. At the first transmission attempt, $C W$ is set equal to the minimum contention window $\left(C W_{\min }\right)$. After each unsuccessful transmission, $C W$ is doubled until it reaches the maximum contention window $\left(C W_{\max }\right)$.

When the destination station successfully receives a frame, it will transmit an acknowledgment frame (ACK) after a short interframe space (SIFS). If the sender does not receive the ACK within a specified ACK timeout, or it detects the transmission of a different frame on the channel, it reschedules the frame transmission according to the previous backoff rules.

DCF also defines an optional four-way handshaking method for frame transmission, which is known as RTS/CTS method. When a station is ready for a transmission, it performs the previous backoff technique and then transmits a special RTS frame. When the receiver receives the RTS frame, it responds with CTS frame after an SIFS. The sender may then transmit the frame only if the CTS frame is correctly received.

The RTS and CTS frames carry the information of the length of the frame to be transmitted, which is used to update a network allocation vector (NAV) by other stations. The NAV contains the information about the period of time in which the channel will remain busy; hence, a station can delay transmission by detecting either RTS or CTS to avoid collisions.

\section{B. Enhanced Distributed Channel Access}

EDCA is specified to provide QoS support on IEEE 802.11 WLANs. It supports up to four access categories (AC), from the lowest priority service class, $\mathrm{AC}_{0}$, to the highest priority service class, $\mathrm{AC}_{3}$. EDCA differentiates service classes through three mechanisms: contention window size, AIFS, and transmission opportunity (TXOP) limit differentiations.

Stations that use smaller $C W_{\min }$ and $C W_{\max }$ receive higher QoS than the other stations as their channel access delays are generally shorter. In EDCA, high priority service class uses smaller $C W_{\min }$ and $C W_{\max }$ to improve the QoS received by the higher priority classes.

In EDCA, AIFS is used instead of DIFS, where AIFS $\geq$ DIFS. Each service class can use different AIFS value to differentiate the QoS received by the service class. Stations that use lower AIFS encounter fewer collisions and count down the backoff counter faster than the other stations; hence, they receive better QoS.

EDCA also allows stations to transmit multiple frames without contending again, known as contention free bursting (CFB). CFB is limited by the TXOP limit specified for each service class. Longer limit means that the service class can transmit more frames; hence, it receives better QoS.

In EDCA, each station implements a queue for each AC. Each queue has its own QoS parameters and backoff counter. A collision within a station is handled virtually, whereby the frame from the highest priority queue involved in the collision is chosen and transmitted to the access medium. This mechanism is known as virtual collision.

\section{RESULTS FROM SATURATION MODEL OF DCF}

In [3], Bianchi uses Markov Chain to model the saturation performance of IEEE 802.11 DCF. This work produces simple equations, from which we can easily calculate the saturation throughput. Xiao [9] has developed a saturation model that considers service differentiation, but this model incorporates backoff freezing mechanism, which actually requires more elaborate modeling [10]. To keep the explanation simple, we use the original Bianchi's assumptions in this paper. Modifications to incorporate backoff freezing and other service differentiation schemes are easy to implement without modifying the method for the non-saturation analysis described in this paper. Therefore, in this section, we describe the extension to the Bianchi's model to incorporate contention window differentiation without the backoff freezing mechanism.

Let $n_{c}$ be the number of stations of class $c$ for $c=0,1,2,3$. Define probability $\tau_{c}$ as the probability that a station of class $c$ transmits in a randomly chosen slot. The probability $\tau_{c}$ is given by [3], [9]

$$
\tau_{c}=\frac{2\left(1-2 p_{c}\right)}{\left(1-2 p_{c}\right)(W+1)+p_{c} W\left(1-\left(2 p_{c}\right)^{m}\right)} .
$$

The probability $p_{c}$ is the probability that a frame from a station of class $c$ encounters a collision on the channel; this probability is computed by

$$
p_{c}=1-\left(1-\tau_{c}\right)^{n_{c}-1} \prod_{i \neq c}\left(1-\tau_{i}\right)^{n_{i}} .
$$

The probabilities $\tau_{c}$ and $p_{c}$ form a set of non-linear equations, which can be solved numerically.

With probability $\tau_{c}$, we can compute the probability $P_{S}^{(c)}$ that a slot contains a successful transmission of frame of class c. Probability $P_{S}^{(c)}$ is given by

$$
P_{S}^{(c)}=n_{c} \tau_{c}\left(1-\tau_{c}\right)^{n_{c}-1} \prod_{i \neq c}\left(1-\tau_{i}\right)^{n_{i}} .
$$

The probability $P_{I}$ that a slot is empty is computed using

$$
P_{I}=\prod_{c}\left(1-\tau_{c}\right)^{n_{c}} .
$$


Define $P_{S}$ as the probability that a slot contains a successful transmission of any class; $P_{S}$ is given by

$$
P_{S}=\sum_{c} P_{S}^{(c)} .
$$

The probability $P_{C}$ that a slot contains a collision is given by $1-P_{I}-P_{S}$.

Now we calculate the service rate of the system for frames from each class. The service rate $\mu_{c}$ for class $c$ is given by

$$
\mu_{c}=\frac{P_{S}^{(c)}}{P_{I} \sigma+P_{S} E\left[T_{S}\right]+P_{C} E\left[T_{C}\right]} .
$$

The variable $\sigma$ is the idle slot duration. The duration of successful frame transmission, $T_{S}$, and collision, $T_{C}$, for the basic and RTS/CTS methods are given by

$$
\begin{aligned}
T_{S}^{b a s}= & H+P+S I F S+\delta+A C K+D I F S+\delta, \\
T_{C}^{b a s}= & H+P+D I F S+\delta, \\
T_{S}^{r t s}= & R T S+S I F S+\delta+C T S+S I F S+\delta+H \\
& +P+S I F S+\delta+A C K+D I F S+\delta, \\
T_{C}^{r t s}= & R T S+D I F S+\delta,
\end{aligned}
$$

where $H$ is the header transmission duration and $P$ is the frame transmission duration. The total service rate of the system, $\mu$, is given by

$$
\mu=\sum_{c} \mu_{c}
$$

\section{ANALYSis OF PROTOCOL SERVICE TIME}

In this section, we analyze the protocol service time of a certain service class i.e. the period between transmissions of a frame by any stations of a service class as seen by the channel. We consider the RTS/CTS mechanism in this section ${ }^{1}$. The implication of this consideration is that the value of $T_{C}$ is constant i.e. $\operatorname{Var}\left(T_{C}\right)=0$.

Let $\theta_{n}^{(c)}$ be the protocol service time of class $c$ for $c=$ $0,1,2,3 ; n$ is the set of the number of stations of each class, i.e. $n=\left\{n_{0}, n_{1}, n_{2}, n_{3}\right\}$. The expectation of $\theta_{n}^{(c)}$ can be computed from the service rate by $\frac{1}{\mu_{c}}$, which gives

$$
E\left[\theta_{n}^{(c)}\right]=E\left[T_{S}\right]+\frac{P_{I} \sigma+P_{C} T_{C}+P_{S}^{(\phi)} E\left[T_{S}\right]}{P_{S}^{(c)}} .
$$

The expectation $E\left[T_{S}\right]$ is the mean of the successful transmission duration, $\sigma$ is the idle slot duration, and $T_{C}$ is the duration of a collision. The probability $P_{S}^{(c)}$ is the probability that a slot contains a successful transmission of frame of class $c$, which is given by (3), and $P_{S}^{(\phi)}$ is the probability that a slot contains a successful transmission of frame of other classes, which is given by $1-P_{I}-P_{C}-P_{S}^{(c)}$.

To study the variance of $\theta_{n}^{(c)}$, we first define $A$ to be the first event to occur on the channel after a successful transmission of class $c$. Four possible events can happen after a successful transmission of class $c$ : an idle slot ( $A=$ idle), a

\footnotetext{
${ }^{1}$ The formula for basic access method can be easily derived with similar approach.
}

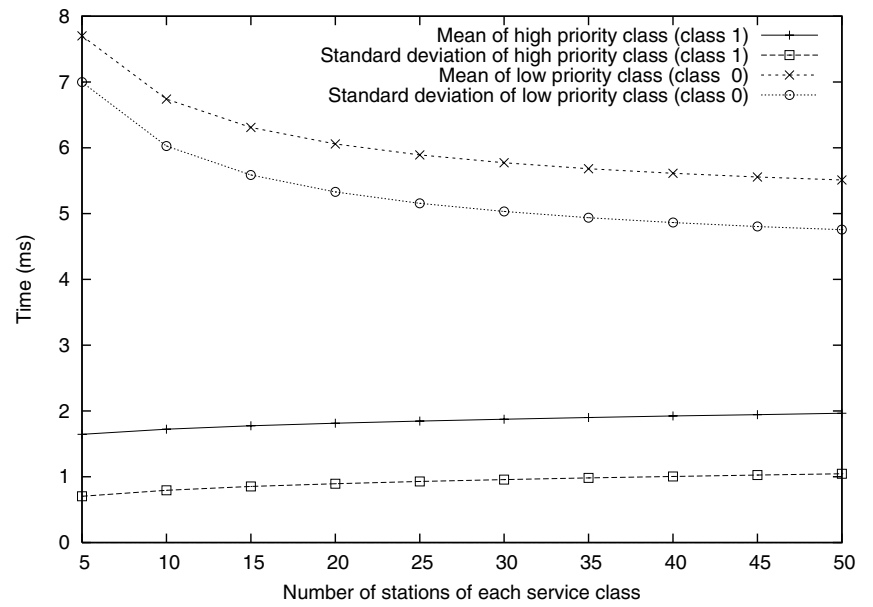

Fig. 1. Mean and variance of the protocol service time.

collision $(A=$ coll), a successful transmission from stations of class $c\left(A=\operatorname{succ}_{c}\right)$, and a successful transmission from the other stations $\left(A=\operatorname{succ}_{\phi}\right)$. We derive the variance of $\theta_{n}^{(c)}$ using the condition variance formula:

$$
\operatorname{Var}\left(\theta_{n}^{(c)}\right)=E\left[\operatorname{Var}\left(\theta_{n}^{(c)} \mid A\right)\right]+\operatorname{Var}\left(E\left[\theta_{n}^{(c)} \mid A\right]\right) .
$$

Notice that $\theta_{n}^{(c)}$ can be divided into two independent random variables: the duration of the first event after a successful transmission of class $c, \nu_{A}$, and the remaining time, $\psi_{A}$; hence, we have $\operatorname{Var}\left(\theta_{n}^{(c)}\right)=\operatorname{Var}\left(\nu_{A}\right)+\operatorname{Var}\left(\psi_{A}\right)$. Owing to the memoryless property of the system, the duration of $\psi_{A}$ itself is a service time i.e. $\psi_{A}=\theta_{n}^{(c)}$. Using this analysis, we obtain the following:

$$
\left\{\begin{array}{l}
\operatorname{Var}\left(\theta_{n}^{(c)} \mid A=i d l e\right)=\operatorname{Var}\left(\theta_{n}^{(c)}\right) \\
\operatorname{Var}\left(\theta_{n}^{(c)} \mid A=\operatorname{coll}\right)=\operatorname{Var}\left(\theta_{n}^{(c)}\right) \\
\operatorname{Var}\left(\theta_{n}^{(c)} \mid A=\operatorname{succ_{c}}\right)=\operatorname{Var}\left(T_{S}\right) \\
\operatorname{Var}\left(\theta_{n}^{(c)} \mid A=\operatorname{succ}_{\phi}\right)=\operatorname{Var}\left(\theta_{n}^{(c)}\right)+\operatorname{Var}\left(T_{S}\right) .
\end{array}\right.
$$

Using these relationships, we have

$$
\begin{aligned}
E\left[\operatorname{Var}\left(\theta_{n}^{(c)} \mid A\right)\right]= & \left(P_{C}+P_{I}+P_{S}^{(\psi)}\right) \operatorname{Var}\left(\theta_{n}^{(c)}\right) \\
& +P_{S} \operatorname{Var}\left(T_{S}\right) .
\end{aligned}
$$

With similar argument for the value of expectation, we have

$$
\left\{\begin{array}{l}
E\left[\theta_{n}^{(c)} \mid A=\text { idle }\right]=\sigma+E\left[\theta_{n}^{(c)}\right] \\
E\left[\theta_{n}^{(c)} \mid A=\text { coll }\right]=T_{C}+E\left[\theta_{n}^{(c)}\right] \\
E\left[\theta_{n}^{(c)} \mid A=\text { succ }_{c}\right]=E\left[T_{S}\right] \\
E\left[\theta_{n}^{(c)} \mid A=\operatorname{succ}_{\phi}\right]=E\left[T_{S}\right]+E\left[\theta_{n}^{(c)}\right] .
\end{array}\right.
$$

The value $\operatorname{Var}\left(E\left[\theta_{n}^{(c)} \mid A\right]\right)$ can be computed by $E\left[\left(E\left[\theta_{n}^{(c)} \mid A\right]-E\left[\theta_{n}^{(c)}\right]\right)^{2}\right]$, which gives

$$
\begin{aligned}
\operatorname{Var}\left(E\left[\theta_{n}^{(c)} \mid A\right]\right)= & P_{I} \sigma^{2}+P_{C} T_{C}^{2}+P_{S}^{(\phi)} E\left[T_{S}\right]^{2} \\
& +\frac{\left(P_{I} \sigma+P_{C} T_{C}+P_{S}^{(\phi)} E\left[T_{S}\right]\right)^{2}}{P_{S}^{(c)}}
\end{aligned}
$$


TABLE I

PARAMETERS FOR THE CONSIDERED SERVICE CLASSES.

\begin{tabular}{c|c|c}
\hline$c$ & $C W_{\min }$ & $C W_{\max }$ \\
\hline 0 & 32 & 1024 \\
1 & 8 & 64 \\
\hline
\end{tabular}

Substituting (12) into (10), we obtain

$$
\operatorname{Var}\left(\theta_{n}^{(c)}\right)=\frac{\operatorname{Var}\left(E\left[\theta_{n}^{(c)} \mid A\right]\right)}{P_{S}^{(c)}}+\frac{P_{S}}{P_{S}^{(c)}} \operatorname{Var}\left(T_{S}\right),
$$

where the value $\operatorname{Var}\left(E\left[\theta_{n}^{(c)} \mid A\right]\right)$ is given by (14) and the probability $P_{S}$ is given by (5).

Consider two service classes with parameters shown in Table I. Furthermore, we assume that the packet size is constant i.e. $T_{S}$ is fixed and $\operatorname{Var}\left(T_{S}\right)$ is zero. We use the parameters of IEEE $802.11 \mathrm{~b}$ standard [11] with packet size of 8184 bits and data rate of 11 Mbps. Fig. 1 shows the mean and the standard deviation of the protocol service time with the specified number of stations for each access class.

With 20 stations, 10 stations are of high priority service class (class 1 ) and the rest of the stations are of low priority service class (class 0 ), the mean and standard deviation of the service time for class 1 are $1.72 \mathrm{~ms}$ and $0.79 \mathrm{~ms}$ respectively, whereby the mean and standard deviation for class 0 are $6.74 \mathrm{~ms}$ and $6.02 \mathrm{~ms}$ respectively. The variance of low priority service class is higher than the high priority class. This is intuitive as the low priority stations have lower probability to access the channel, which allows many high priority stations to access the channel before them; hence, the low priority stations experience long and high variance service time. The figure also shows that the mean and variance of the protocol service time have little variation across the various number of stations.

\section{ANALYTICAL MODEL FOR EDCA UNDER STATISTICAL TRAFFIC}

In this section, we extend [7] to include service differentiation in EDCA. The results in [7] show that we can model DCF protocol with a M/PH/1 single server queue. We use the similar approach whereby we model the protocol service time with phase type distribution.

Similar to [7], we model the access medium, i.e. the wireless channel, as a server that serves the frame transmissions by the stations. As EDCA provides service differentiation, we use multiple servers to model the access medium; each of these servers serves only a service class. The arrival rate to the system is the aggregated arrival rate of the idle stations as observed by the access medium. We assume that the arrival process of the stations is Poisson and the stations can only keep one data frame in their local MAC buffers; thus, we can model the system arrival process as Poisson process. The combined service rate of these servers is the protocol service rate; hence, the service rate of each server is a proportion of the total service rate and depends on the combinations of the number of stations of each class in the queues of the servers ${ }^{2}$.

The mean service rate of a server for service class $c$ is the protocol service rate for the corresponding service class under saturation, which is given by (6). To accurately model the protocol service, we use the results of variance analysis from the previous section.

Considering the same 20 stations scenario as in the previous section, the mean and standard deviation of the service time for class 1 are $1.72 \mathrm{~ms}$ and $0.79 \mathrm{~ms}$ respectively, which correspond to Erlang distribution with five exponential stages; the mean and standard deviation for class 0 are $6.74 \mathrm{~ms}$ and $6.02 \mathrm{~ms}$ respectively, which correspond to an exponential distribution. From previous section, we know that the mean and variance of the protocol service time do not change much (see Fig. 1). To simplify the system model, we fix the number of Erlang stages to five and one (exponential distribution) for class 1 and class 0 respectively. Clearly this in an approximation, but we will show that this approximation will still yield good results since the mean and variance of the protocol service time have little variation. With those considerations, we model the access medium with two servers: one having Erlang distributed service time, and the other having exponential distributed service time.

Let system state $\left\{s_{0}, s_{1}, j\right\}$ shows that the system currently contains $s_{0}$ stations of class 0 and $s_{1}$ stations of class 1 , and the service for class 1 is in the $j$-th stage. Furthermore, let the invalid states of the Markov Chain model, i.e. state $\left\{s_{0}, s_{1}, j\right\}$ outside the range of $0 \leq s_{x} \leq n_{x}$ for $x=0,1$ and $1 \leq j \leq \omega$, has stationary probability of zero. The variable $\omega$ is the number of Erlang stage for class 1. We also assume that the arrival and service rates are zeros for out-of-range parameters i.e. arrival rate is non-zero if $0 \leq \lambda_{c}<n_{c}$ and service rate is non-zero if $0<\mu_{c} \leq n_{c}$. The balance equations for the system are given by

$$
\begin{aligned}
0= & -\left(\lambda_{0}\left(s_{0}\right)+\lambda_{1}\left(s_{1}\right)\right. \\
& \left.+\mu_{0}\left(s_{0}, s_{1}\right)+\omega \mu_{1}\left(s_{0}, s_{1}\right)\right) b_{s_{0}, s_{1}, 0} \\
& +\lambda_{0}\left(s_{0}-1\right) b_{s_{0}-1, s_{1}, 0}+\lambda_{1}\left(s_{1}-1\right) b_{s_{0}, s_{1}-1,0} \\
& +\mu_{0}\left(s_{0}+1, s_{1}\right) b_{s_{0}+1, s_{1}, 0}+\omega \mu_{1}\left(s_{0}, s_{1}+1\right) b_{s_{0}, s_{1}+1, \omega} \\
0= & -\left(\lambda_{0}\left(s_{0}\right)+\lambda_{1}\left(s_{1}\right)\right. \\
& \left.+\mu_{0}\left(s_{0}, s_{1}\right)+\omega \mu_{1}\left(s_{0}, s_{1}\right)\right) b_{s_{0}, s_{1}, m} \\
& +\lambda_{0}\left(s_{0}-1\right) b_{s_{0}-1, s_{1}, m}+\lambda_{1}\left(s_{1}-1\right) b_{s_{0}, s_{1}-1, m} \\
& +\mu_{0}\left(s_{0}+1, s_{1}\right) b_{s_{0}+1, s_{1}, m}+\omega \mu_{1}\left(s_{0}, s_{1}\right) b_{s_{0}, s_{1}, m-1},
\end{aligned}
$$

with the normalization condition

$$
\sum_{s_{0}=0}^{n_{0}} \sum_{s_{1}=0}^{n_{1}} \sum_{j=1}^{\omega} b_{s_{0}, s_{1}, j}=1
$$

\footnotetext{
${ }^{2}$ The variable service rate is caused by the changes in service rate of the access medium with the changes of the number of stations being served [7].
} 
These equations can be solved numerically, which yields the state's stationary probability $b_{s_{0}, s_{1}, j}$. Let $P_{x}^{(c)}$ denotes the stationary probability of the system having $x$ stations of class $c$; this probability can be computed by

$$
\begin{aligned}
& P_{x}^{(0)}=\sum_{i=0}^{n_{1}} \sum_{j=1}^{\omega} b_{x, i, j} \\
& P_{x}^{(1)}=\sum_{i=0}^{n_{0}} \sum_{j=1}^{\omega} b_{i, x, j} .
\end{aligned}
$$

The mean arrival rate for class $c$ as observed by the system, $\bar{\lambda}_{c}$, is given by

$$
\bar{\lambda}_{c}=\sum_{x=0}^{n_{c}}\left(\lambda_{c}(x) \cdot P_{x}^{(c)}\right) .
$$

The aggregated throughput of the stations from each service class, $\rho_{c}$, can be computed from $\bar{\lambda}_{c}$ by

$$
\rho_{c}=\bar{\lambda}_{c} \cdot P,
$$

where $P$ is the mean transmission time of the payload in the data frame.

The mean delay of a frame transmission from a station of class $c, D^{(c)}$, is computed using Little's theorem:

$$
D^{(c)}=\bar{n}_{c} / \bar{\lambda}_{c}
$$

where $\bar{n}_{c}$ is the average number of stations of class $c$ in the system, which is given by $\sum_{x=0}^{n_{c}}\left(x P_{x}^{(c)}\right)$.

\section{RESUlTS AND DisCUSSIONS}

We run experiments with 10 stations of class 0 and 10 stations of class 1 for a total of 20 stations. Fig. 2 shows the aggregated throughput of each service class comparing the analytical results (the solid lines) to the simulation results (the symbols). Our simulations follow the assumptions in [3]. The arrival model for the simulations is Poisson process and each station can keep only one data frame in its local MAC buffer. The figure shows close match between analytical and simulation results. In this figure, we also observe that EDCA provide throughput differentiation; the high priority stations have larger bandwidth share. This differentiation is not observed on low arrival scenario as the bandwidth is enough for all classes. Note that this throughput differentiation characteristic was demonstrated with simulations in [12].

Fig. 3 shows the delay of a frame transmission ${ }^{3}$ from a station of each service class. This figure shows the evidence of delay differentiation capability of EDCA mechanism. The delay differentiation is observed even at low arrival rates, which is intuitive as the high priority stations can transmit after shorter waiting period caused by contention window. The mean delay of high priority stations is kept below $20 \mathrm{~ms}$ even on high arrival rates with the expense of high delay for the low priority stations.

\footnotetext{
${ }^{3}$ Delay measure collected from the simulation is the period from when a data frame reaches a local buffer until it is successfully received by the receiver
}

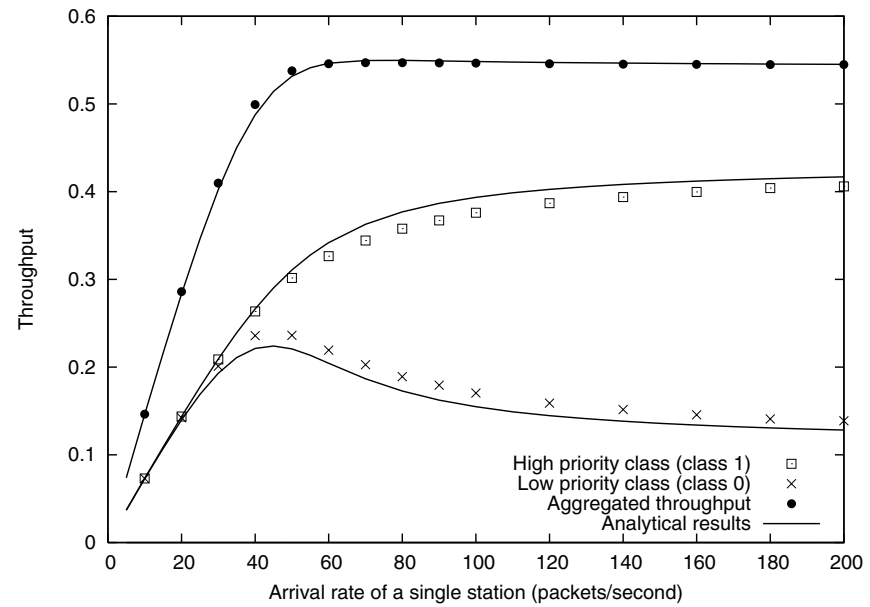

Fig. 2. Throughput for 10 stations of each service class.

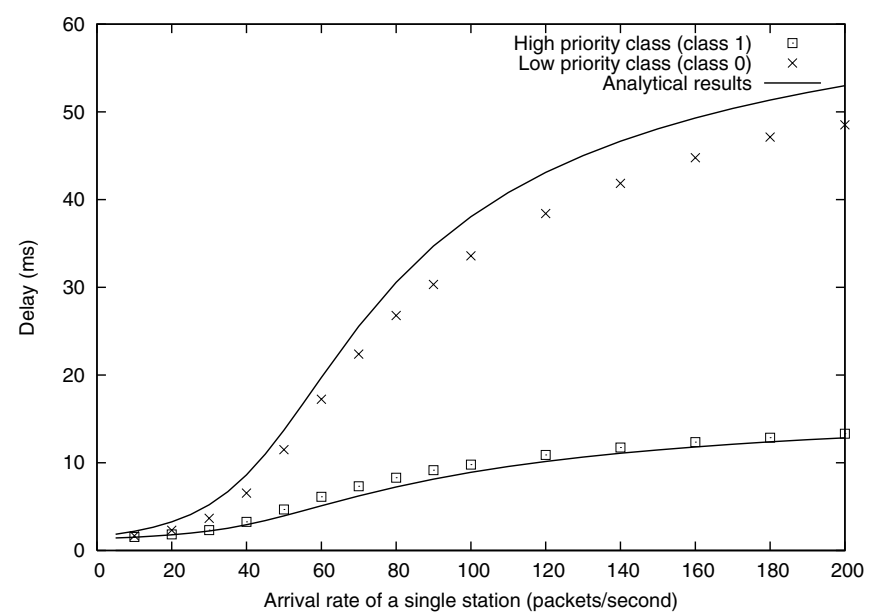

Fig. 3. Delay for 10 stations of each service class.

In Fig. 4, we plot the aggregated throughput versus delay for each service class. As the throughput of the high priority stations grows, the throughput of the low priority stations decreases and the delay of the low priority stations grows fast. The figure shows that the high priority stations can obtain a significant portion of the bandwidth (up to $75 \%$ of the total throughput) while maintaining a delay lower than $20 \mathrm{~ms}$.

\section{CONCLUSION}

In this paper, we have analyzed the protocol service time of EDCA mechanism and introduced a model to analyze EDCA under statistical traffic using Markovian Framework. The analysis of the protocol service time is important to select an accurate parameter for the Markovian Framework model. The coefficient of variation is shown to be different for different class; hence, we use different service model for different class in the modeling. Using this model, we analyzed the throughput and delay performance of EDCA mechanism under statistical 


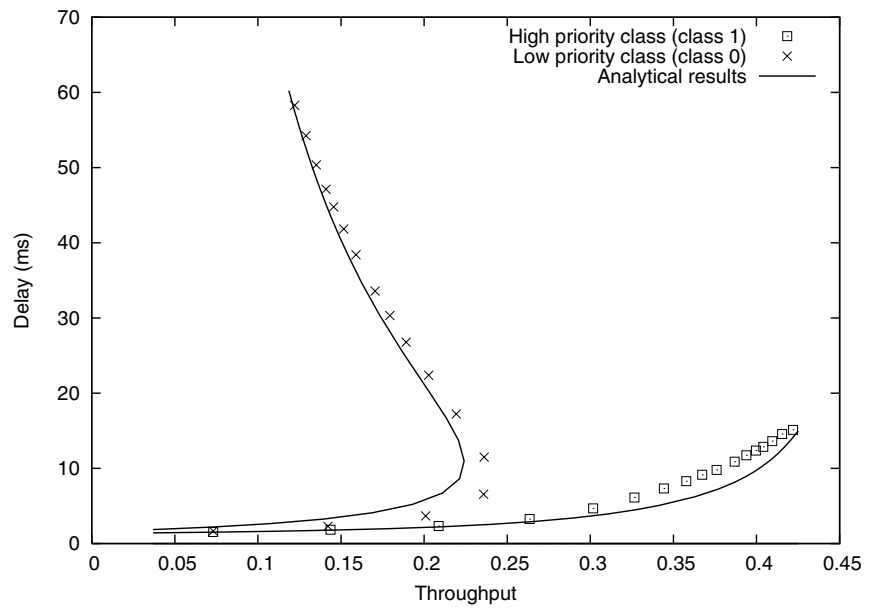

Fig. 4. Throughput versus delay with 10 stations of each service class.

traffic. The results show the evidence that EDCA does provide service differentiation under statistical traffic condition, which is visible in the difference of throughput and delay for different service class.

Our model is useful to predict and analyze the performance of the WLANs that implement the IEEE 802.11 standards. Another application is for network sizing (a method is discussed in [8], which uses the Markovian Framework introduced by Foh and Zukerman).

\section{REFERENCES}

[1] Wireless LAN Medium Access Control (MAC) and Physical Layer Specification, IEEE Std. 802.11, 1997.

[2] Draft Supplement to Part 11: Wireless Medium Access Control (MAC) and Physical Layer Specification: Medium Access Control (MAC) Enhancements for Quality of Service (QoS), IEEE 802.11 WG Std. 802.11e, 2002.

[3] G. Bianchi, "Performance analysis of the IEEE 802.11 distributed coordination function," IEEE J. Select. Areas Commun., vol. 18, no. 3 , pp. 535-547, Mar. 2000.

[4] P. Chatzimisios, A. C. Boucouvalas, and V. Vitsas, "IEEE 802.11 packet delay - a finite retry limit analysis," in Proc. IEEE Globecom, San Francisco, USA, Dec. 2003.

[5] J. W. Robinson and T. S. Randhawa, "Saturation throughput analysis of IEEE 802.11e enhanced distributed coordination function," IEEE $J$. Select. Areas Commun., vol. 22, no. 5, pp. 917 - 928, June 2004.

[6] J. W. Tantra, C. H. Foh, and A. B. Mnaouer, "Throughput and delay analysis of the IEEE 802.11e EDCA saturation," in Proc. IEEE ICC, Seoul, Korea, May 2005.

[7] C. H. Foh and M. Zukerman, "Performance analysis of the IEEE 802.11 MAC protocol," in Proc. European Wireless 2002, Florence, Italy, Feb. 2002.

[8] P. Patil and V. Apte, "Sizing of IEEE 802.11 wireless LANs," in Proc. ACM WMASH, Cologne, Germany, Sept. 2005.

[9] Y. Xiao, "A simple and effective priority scheme for IEEE 802.11," IEEE Commun. Lett., vol. 7, no. 2, pp. 70-72, Feb. 2003.

[10] C. H. Foh and J. W. Tantra, "Comments on IEEE 802.11 saturation throughput analysis with freezing of backoff counters," IEEE Commun. Lett., vol. 9, no. 2, pp. 130-132, Feb. 2005.

[11] Part 11: Wireless LAN Medium Access Control (MAC) And Physical Layer (PHY) Specifications: High-speed Physical Layer Extension In The $2.4 \mathrm{GHz}$ Band, IEEE Std. 802.11b, Sept. 1999.

[12] S. Mangold, S. Choi, G. R. Hiertz, O. Klein, and B. Walke, "Analysis of IEEE 802.11e for QoS support in wireless LANs," IEEE Wireless Commun. Mag., vol. 10, no. 6, pp. 40-50, Dec. 2003. 